\title{
On the Selection and Layout of Advanced Mathematics Course Content
}

\author{
Duan Wenxi \\ School of Applied Mathematics, Beijing Normal University Zhuhai, China \\ 236304613@qq.com
}

\begin{abstract}
The selection and layout of advanced mathematics should focus on the basics, decrease theorem proving and derivation of the formula and increase the application of knowledge, delete abstract theories, slightly adjust the order of the content and reflect its professional features. The strict definition of the limit should be deleted while its descriptive definition should be supplemented.

Index Terms - Advanced mathematics, selection and layout of content, limit theory
\end{abstract}

\section{Introduction}

Schools of higher education refer to schools providing higher education for the citizens. More specifically, they refer to institutions of higher education which provide conditions for teaching and research, and are authorized to issue a certificate of degree. Schools of higher education also include College. Speaking from the type of school, schools of higher education include colleges and universities, adult colleges, private colleges and universities and so on.

The main difference between general education and adult education is that the objects of education and forms of learning are different. General education is intended primarily for young people in the growth stage, and it takes the form of full-time teaching. Obviously, the objects of adult education are adults and it is generally part-time teaching

General higher education generally refers specifically to the education of undergraduates and junior college students. The former is organized by universities, undergraduate colleges and independent colleges, and the latter is organized mainly by vocational and technical colleges and advanced junior colleges. Universities, undergraduate colleges and independent colleges also undertake some mission of professional training. Vocational and technical colleges and advanced junior colleges are not authorized to issue a certificate of undergraduate academic qualifications.

In China, schools of adult higher education refer to schools that recruit staff and workers of high school or equivalent education as the main training object through the National Adult Higher unified entrance examination (Adult college entrance examination) and teach them in forms of correspondence, part-time and full-time, in accordance with national regulations setting standards and approval procedures. They include radio and TV university, the university of workers, amateur university, medical college, management college, education colleges and adult (continuation) education colleges in general universities.

In China, different type of education has different definition on advanced mathematics. Institutions of higher education integrate mathematical analysis, analytic geometry with ODE and name it advanced mathematics; while in selfstudy examination of higher education and graduate record examination, the aforementioned higher mathematics, linear algebra, probability theory and mathematical statistics are known as higher mathematics. What is mentioned in this article refers to the former.

Advanced mathematics is a public compulsory course for those who major in engineering, economic and management in institutions of higher education, and it is also the basis for subsequent professional courses. The selection and layout of their contents is an important factor affecting the quality of teaching, so we should attach great importance to the selection and layout of higher mathematics curriculum.

\section{Focusing on the Basics and Decreasing Reasoning and Proving}

The teaching plan of advanced mathematics is usually characterized by few class hours but much content. But it is unrealistic to lecture everything in detail in a limited period of times, therefore, it can only focus on teaching basic definitions, fundamental theorem, the basic formula and basic methods. It is advisable to decrease the theoretical proving. The proving of classic theorem and those are helpful to solve problems should be instructed and demonstrated, but do not deduce every theorems and formulas.

For example, when introducing two important limits, we should focus on how to identify and calculate them and eliminate their derivation and proving. In the teaching of continuous function, the concept of continuous function and related theorems should be introduced, but not the proving of intermediate value theorem; When introducing the derivative and differential, we should focus on their concepts and emphasize both their relations and distinctions; Another example is that when lecturing on the properties of differential mean value theorem, the definite integral and the like, the explanation should be from the geometric aspect and never spend a lot of time to prove them. The most important thing is to illustrate the application of these theorems and their properties.

In addition to diluting the proving of theorems and derivation of the formulas, complex calculations should also be diluted. For example, the indefinite integral is the basis of definite integrals, and the main purpose of introducing indefinite integrals is to calculate the definite integral, but now computer softwares have been developed to calculate the definite integral and it is easy to calculate by using these 
softwares, so we do not need to teach the calculation of indefinite integral as a key content.

It is unrealistic to require the students to quickly grasp a lot of theoretical knowledge in a short period of time without a digestion process. For non- math majors, if they can remember the above four "basic", use them to solve problems and apply them into practice, they have reached the ultimate goal of teaching .

\section{Deleting the Unnecessary}

Textbooks of advanced mathematics adopted by colleges and universities around the world are similar in their content and the focus. The content and layout systems follow the characteristics of materials of the $50 \mathrm{~s}$ and 60 s of last century, and there is no major change until now. With the continuous development of science and technology, social reality has put forward new demands on mathematics, therefore, it is necessary to appropriately change the content and layout of higher mathematics so as to adapt to people's actual needs of mathematics .

The contents that need deleting include the following parts:

\section{A. The repetitive content}

An example is that we have learned the concept of set in high school, therefore, it is unnecessary to repeat it in advanced mathematics textbooks.

\section{B. The purely theoretical content}

For example, the " $\delta-\varepsilon$ language" theory of limit [1], beginners find it difficult to learn, and there is no practical value in the process of getting the limit, so it should be deleted completely. After removing the this content about limit, an intuitive description [2] towards the limit is the most appropriate.

\section{Ways of solving problems with too much skills}

For example, the calculation of triangle rational fraction in indefinite integral should be deleted and the reason is that with the continuous development of computer knowledge, you can easily calculate these

\section{Adding the Necessary}

In order to make an orderly convergence around the content and reflect its gradual, scientific and applicable characteristics, we need to appropriately add relevant content.

The main contents that need complementing include the following aspects

\section{A. Supplementing the concept of piecewise function.}

The characteristics of piecewise function and the differences between it and elementary functions;

\section{B. Supplementing the concept of polar coordinates.}

When calculating the double integral polar coordinates, the concept is needed but it is not introduced in some textbooks, so it is needed to make appropriate supplements in advanced mathematics textbook, and describe the representation form of common curve in Cartesian coordinates, polar coordinates and parametric equations.

\section{Supplementing infinitesimal equivalent substitution formula}

The use of infinitesimal equivalence to get limit is an ingenious problem-solving approach. Under certain conditions, the use of this method is even simpler that the use of L'Hospital's rule, therefore, in the introduction of method to get limit, we should add infinitesimal equivalent substitution formula [3] ; and introduce how to use these formulas to easily get the limit.

\section{Supplementing the derivative method of piecewise functions} [4]

Students tend to mistake the derivative of elementary functions for the derivative of piecewise functions, therefore, in order to make sure that students can distinguish them, descriptions and examples of the derivative method of piecewise functions should be added.

In the definite integral, examples of the concept of maximum change of definite integral and its properties should be added; however, the add and deletion should not be so much that it becomes away from the students and beyond their acceptable scope .

\section{Adjust the Content Sequence}

The contents of Mathematics are closely related in a cohesive, orderly, progressive, and highly systematic way. When teaching, we should take advantage of these features, but if we are not flexible, it is not necessarily to get good teaching effect. To enable students to learn the content by regarding them as blocks, we must adjust the order of chapters or contents, otherwise, students may feel that there is no logic in the textbook, and students can not easily sort out the contents and memorize them.

For example, continuation in advanced mathematics is a concept defined by limit, therefore, the concept of continuation should be introduced after the concept of limit has been fully introduced. But in reality, in the existing materials the concept of continuation is introduced before the limit of elementary function (e.g: $\left.\lim _{x \rightarrow 0} \frac{\ln (1+x)}{x}=1, \lim _{x \rightarrow 0} \frac{e^{x}-1}{x}=1.\right)$. This layout system, although can ensure the rigor and logic of mathematics, but in the actual teaching and learning, it can't achieve good results, because such layout is not easy for students to summarize and memorize in blocks. Therefore, we propose to reverse the order of their teaching, that is, at first, we demonstrate that elementary functions can be directly substituted to get the limit in a meaningful point and after finishing the lecture of the continuity of the function, we explain the reasons for this is that an elementary function is continuous in meaningful points.

Here is another example. The layout of the existing textbooks on derivatives is organized as follows : After introducing the definition of the derivative, the power functions, exponential functions, logarithmic functions, the sine function, and cosine function derivative formulas are deduced; After introducing the deducing method of inverse function, inverse trigonometric derivative formulas are deduced; After the introduction of four arithmetic operations 
of derivatives, tangent and cotangent derivative formulas are deduced. That is to say, in the existing materials, the introduction of the concept of derivatives and related theorems come first and at last all of the derivative formula are summed up .Such layout system dilutes the importance of the derivative formula, resulting in a wrong impression on students that the theorems of the derivatives are more important than the formulas themselves.

In fact, the derivative formula is the most important mathematics formula, which is the basis of differential and integral equations. Therefore, it is proposed that after the introduction of derivative concept, all the derivative formula should be immediately given, requiring students to well remember these formulas, apply them to get the derivative of elementary function, and then gradually show the origin and derivation of the above formula through the guide of a relevant theorem.

The adjustment of the order of the content should follow the principles of fine-tuning, and the content should not be largely inverted. If textbooks talk about the definite integral, and then the indefinite integral, the adjustment is unscientific. That is because when calculating the definite integral by using Newton - Leibniz formula, the plot function of the original function is used, and the set of the original function is indefinite integral, therefore, the scientific way is to talk about indefinite integral, and then the definite integral.

It needs noting that it is not advisable to arbitrarily reverse the order of courses, for example, a large part of probability theory and mathematical statistics involves the calculation of the definite integral and double integral calculations, and statistical component also involves the partial derivative and least squares method. Therefore, probability theory and mathematical statistics should be opened after advanced mathematics.

\section{Highlighting Professional Features and Emphasizing Application}

The content of advanced mathematics should reflect professional features and adapt to them. In the major of engineering, the concept of a periodic function is frequently present in the subsequent circuit analysis, mechanical engineering, automatic control and other courses, therefore, the content of periodic functions should be added in the textbooks for engineering major. In the major of economics, the follow-up courses involve economics, so the textbooks for economics student should highlight the applications of mathematics in economics.

Some of the content is with clear professional features, and can only be present in their respective textbooks. For example, the marginal function, the function of the elasticity and the like are concepts in economics [5], and they should be present in the textbook for economic students; while the line integrals, surface integrals, Fourier series are engineering issues and they should be in teaching materials for engineering students.

\section{The Lecture of Limit Theory}

The first chapter of advanced mathematics content is the conception of function, which main content is to introduce the concept of function extremely nature -based, high school students have long been studied in the content they already have on the concept of perceptual function, therefore, when students at the University of repetitive learning function, they generally feel easy to accept . But according to the teaching plan, the contents of the function up to arrange four hours , therefore, before entering college students are learning state, the teacher began teaching limit theory.

Limit concept is the cornerstone of higher mathematics , higher mathematics, the main concepts are expressed through extreme forms, therefore, limit concept is the basic principle of higher mathematics. The importance of the concept of limit, it is not only reflected in the foundation underwent content, and more importantly, when the teacher taught the limit period, most students enter college early, rapid changes in the teachers guide students to learn ways to adapt to university learning environments a critical period, during this period, if students understand the university's learning characteristics, mastered mathematics learning methods, then the student will ease up learning content, but if the teacher during this period handle improperly with teaching methods teaching contrary to the law, students will be completely misled, resulting in students' incorrect understanding of the university, developing the wrong study habits.

Currently, both the science and engineering professionals to use advanced mathematics textbook, or economic management professional use calculus textbook, on the presentation of the concept of limit, they widely use the " $\delta-\varepsilon$ language" form. This definition is strictly defined limits. Grasp this definition, not only to lay the mathematical foundation for further study of a high -level math courses pave the way, the main train students to be able to abstract thinking skills. This definition of the limits of the following two main features:

A .Abstractness: Students not easy to understand the meaning of the definition and do not understand the relationship between them, they do not understand the link between the limits.

B. scaling resistance: Students seeking the limits of the process, you want to zoom in or out mathematical equations to find the relationship. For just entering college students, this requirement is a completely new and unfamiliar mathematical Thinking method. They can't be immediately used to and accepted this approach. Because of the above two features, students generally feel not easy to understand the limits of this concept. According to the survey, students listen whenever the limit "language", the majority of students do not understand this definition, only $5 \%$ of the students feel understanding superficially, even math majors have such a feeling. We can't say that students always do not understand the limits of the strict definition, when an interim examination after the course, even to the end of the period, the students come to completely aftertaste strictly defined limits, then although understood, but has been on students' learning follow-up content had an impact, these effects mainly in the following aspects: 
(1) Makes the students believe that mathematics is the essence of the derivation of mathematical formulas and transformations, there is no application value math games;

(2) To the students resulted in higher mathematics difficult to learn, do not understand the teacher's classroom teaching should be, on what they learn confused is normal.

(3) Lead to the wrong students develop study habits, making them think that learning mathematics, is to learn how to make proofs, rather than the most basic knowledge of the memory elements, and ultimately makes the students so that he did not know what the basic concepts of mathematics, basic conclusion, there is no limit exists some very good grasp of proven methods, but do not know mathematics to solve the specific problems in daily life.

(4) Under normal circumstances, the first semester of college freshmen, and most of this semester, two weeks before learning the concept of limit, as strictly defined limits of abstraction, a blow to the self- learning center, resulting in students considered themselves stupid, no ability to learn mathematics, often leads to $10 \%$ of the students will give up mathematics learning .In order to make the students easy to accept, and can lay the foundation for the study follow-up content, we suggest the limits of the teacher taught descriptive definition, so that students can not only easily understand the concept of limit, the limit will also be taught to avoid the strict definition of the teacher brought negative impact. For nonmath majors, the purpose of learning mathematics in the application, therefore, the teacher should seek emphasis on the limits of law and the subsequent application of the content. In fact, scientists have discovered many theorems and formulas, and later the scientists do not know the reason behind these theorems and formulas, but they will apply these results to practical problems, and therefore, to promote the development of science and technology .Beijing Normal University is China 's century-old school, the school teacher has a wealth of experience in teaching, mathematics teachers to make their students successfully learn the concept of limit, they take the first limit taught descriptive definition, and then wait for students to adapt university learning environment, and then re- teach the limits of strict definition. This way of teaching is welcomed by all sessions of students, and the teaching effect is obvious. Math teacher suggests that other math teachers from other colleges and universities can also follow this teaching method.

The precautions the paper states universally can be applied to probability theory and mathematical statistics, linear algebra, and other math lessons, in Mathematics preparation of teaching materials, they can refer to the proposed point of view.

\section{References}

[1] Cao Dinghua, "calculous (in China)" ,Shang Hai: Fudan University press, 2009, pp 19-32

[2] Wen-xi Duan, "Apply for graduate students of Mathematics", Guang dong, Ji Nan University press, 2011, pp 10-11.

[3] Wen-xi Duan, "Apply for graduate students of Mathematics", Guang dong, Ji Nan University press, 2011, pp 16.

[4] Shu-yuan Zhao, "calculous (in China)," Peking: Renmin University of China press, 1987, pp 10-13. 